\title{
Creating Personalised Energy Plans: From Groups to Individuals using Fuzzy C Means Clustering
}

\author{
[Extended Abstract] \\ Ian Dent, Christian Wagner, Uwe Aickelin and Tom Rodden \\ School of Computer Science \\ University of Nottingham \\ Nottingham, UK, NG8 1BB \\ ird, cxw, uxa, tar@cs.nott.ac.uk
}

\begin{abstract}
Changes in the UK electricity market mean that domestic users will be required to modify their usage behaviour in accordance with energy efficiency targets. Clustering allows usage data, collected at the household level, to be clustered into groups and assigned a stereotypical profile which may be used to provide individually tailored energy plans. Fuzzy C Means extends previous work based around crisp K means clustering by allowing a household to be a member of multiple customer profile groups to different degrees, thus providing the opportunity to make personalised offers to the household dependent on their degree of membership of each group. In addition, feedback can be provided on how household's changing behaviour is moving them towards more "green" or cost effective stereotypical usage.
\end{abstract}

\section{Categories and Subject Descriptors}

H.4 [Information Systems Applications]: Miscellaneous

\section{General Terms}

Algorithms, Economics, Human Factors

\section{Keywords}

Electricity load profiles, Clustering, Fuzzy C Means, Demand Side Management

\section{INTRODUCTION}

The electricity market in the UK is under various pressures. Some are due to the history and current design of the National Grid and others are arising from worldwide trends,

\footnotetext{
*Ian Dent is the corresponding author.

Ian Dent, Christian Wagner and Uwe Aickelin are affiliated with the Intelligent Modelling and Analysis Research Group. Ian Dent, Christian Wagner and Tom Rodden are affiliated with the Horizon Digital Economy Research Institute.
}

such as the need to reduce carbon emissions and the declining sources of hydro-carbon fuels. New technologies, such as electric cars needing household charging facilities, will be much more common. The information monitored in the home will grow rapidly, particularly with the roll out of Smart Meters planned for completion in the UK by 2019. In addition, the drive to change the mix of electricity generation technologies in order to reduce greenhouse gas emissions, the desire to reduce carbon dioxide by changing nonelectric demand such as gas central heating to the electricity network, and the impact of climate change on altering electricity demand and the greater occurrence of extreme weather events will increase the difficulties in providing a stable and cost effective supply without better modelling of the patterns of consumption within the grid.

One approach to addressing these pressures is the application of Demand Side Management (DSM) techniques to achieve changes in consumer behaviour at a domestic consumer level [5]. The Desimax project [3] (of which this work forms part) concentrates on applying DSM techniques to achieve better electricity system efficiency.

DSM techniques require an understanding of the spread of electricity usage of each household - the load profile. Once this is known, the most appropriate households to target and the desired changes can be determined (e.g. moving the time of peak demand).

\section{METHODOLOGY}

Clustering techniques (e.g. K Means) allow for the grouping of households into a small number of clusters to which targeted marketing campaigns can be applied. Each household is assigned to one cluster/group/stereotypical profile but, in reality, may show aspects of many clusters. The Fuzzy C Means clustering algorithm [4] allows the allocation of households to many clusters together with a degree to which the household is a member of a cluster.

Investigation has made use of hourly usage data collected from 93 households in Milton Keynes over a period of 2 years. The data is split into winter and summer seasons and into weekdays and weekends. Any days containing missing readings are omitted from the analysis, the data is normalised into the 0:1 range and the mean calculated for each hour of the day. An average reading for each of the 93 house- 
holds is obtained that consists of 24 hourly readings, each of which are calculated by averaging each day over the readings for the winter weekends.

In previous work [1] the application of "crisp" K means and Self Organised Maps algorithms to the Milton Keynes data allows for a set of 9 clusters to be determined, where each of the households is assigned to one of the stereotypical profiles. This paper extends the work using Fuzzy C Means to allow a household to be assigned to multiple stereotypical profiles to differing degrees, allowing a more fine-grained targeting of households and, for example, the creation of individually tailored energy plans.

\section{RESULTS}

The clusters derived from the Fuzzy C Means algorithm are shown at Figure 1. Each household has a degree of membership of each of the clusters where Figure 2 provides an example based on the Milton Keynes data. It indicates the membership of one specific household to extracted usage profiles (clusters) to different degrees. Considering the different degrees of membership, it is clear that a strict association of a household to one and only one usage profile/cluster implies the loss of significant information and prevents an accurate targeting of the specific usage patterns of individual households. This work does not currently focus on the optimum number of clusters (9 were chosen to be comparable with other work), but aims to detail the approach and showcase the principle that households can be members of different profiles to different degrees.

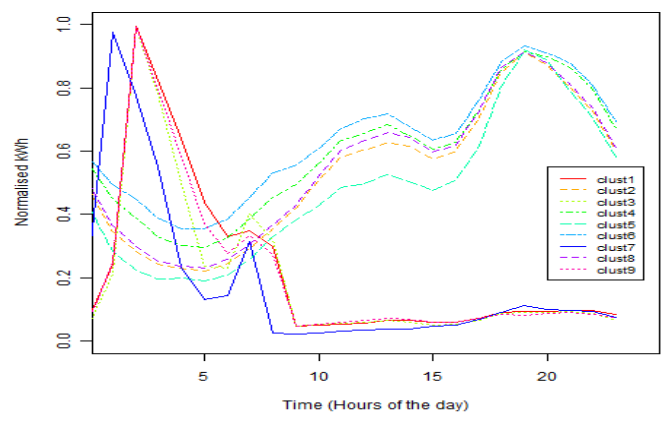

Figure 1: Representative Clusters

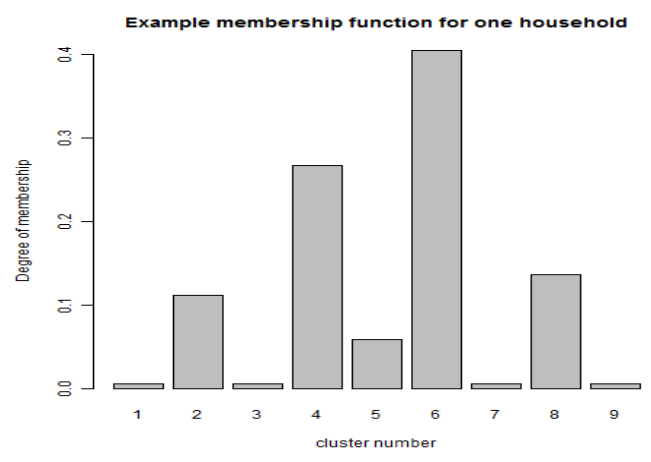

Figure 2: Example cluster membership distribution

\section{CONCLUSIONS}

The suggested approach allows energy suppliers to define offers for each of the 9 stereotypical profiles but to offer their customers individualised tariffs based on their degree of membership of the clusters aligned with the stereotypical profiles.

As an example of how this may be used, the energy supplier may wish to see some movement of usage of electricity for the households similar to "cluster5" from the peak in early evening to the much lower usage around the middle of the day. They may develop a tariff offer for "cluster5" and then offer this to their customers with a weight depending on the household's membership of that cluster. This could be combined with offers for the other clusters such that each household is offered a tariff made up of a personalised (based on cluster membership degrees) "cocktail" of energy-profile specific tariffs.

The results could also be used to feedback to consumers on how their day to day behaviour is changing and how they are gradually moving towards more "green" stereotypical usage patterns in the same way that dieting regimes show success by feedback of gradual short term improvements to the dieters.

\section{ACKNOWLEDGEMENTS}

The Milton Keynes data was accessed through the UK Energy Research Centre Energy Data Centre (UKERC-EDC). Our acknowledgements to the Building Research Establishment, which provided access to the original 1990 data set from Milton Keynes Energy Park, and to Bartlett School of Graduate Studies, University College London for processing and cleaning the raw data. [2] [6]

This work was possible thanks to EPSRC grant references $\mathrm{EP} / \mathrm{I} 000496 / 1$ and EP/G065802/1.

\section{REFERENCES}

[1] I. Dent, U. Aickelin, and T. Rodden. Application of a clustering framework to UK domestic electricity data. In 11th Annual Workshop on Computational Intelligence, 2011.

[2] J. Edwards. Low energy dwellings in the Milton Keynes Energy Park. Energy Management, 26:32-33, 1990.

[3] A. Kiprakis, I. Dent, S. Djokic, and S. McLaughlin. Multi-scale dynamic modeling to maximize demand side management. In IEEE Power and Energy Society Innovative Smart Grid Technologies Europe 2011, Manchester, UK, 2011.

[4] N. Pal, J. Bezdek, and R. Hathaway. Sequential competitive learning and the fuzzy c-means clustering algorithms. Neural Networks, 9(5):787-796, 1996.

[5] C. River. Primer on demand-side management with an emphasis on price-responsive programs. prepared for The World Bank by Charles River Associates, Tech. Rep, 2005.

[6] A. Summerfield, R. Lowe, H. Bruhns, J. Caeiro, J. Steadman, and T. Oreszczyn. Milton Keynes Energy Park revisited: Changes in internal temperatures and energy usage. Energy and Buildings, 39(7):783-791, 2007. 